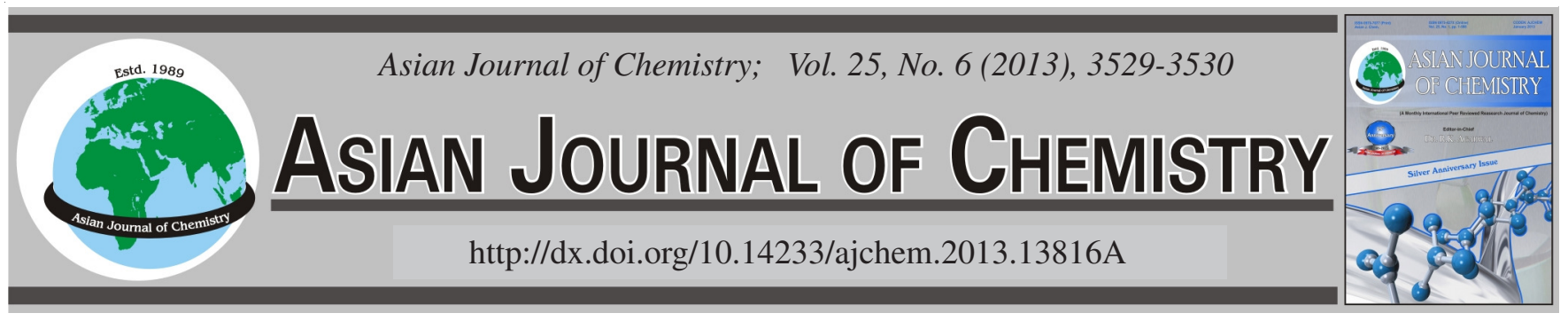

NOTE

\title{
A New Cadmium(II) 2,6-Naphthalene dicarboxylic Acid Three-Dimensional Metal-Organic Frameworks with 6-Connected PCU Topology
}

\author{
SI-CAI XIE \\ Biology and Chemistry Engineering College of Panzhihua University, Sichuan 617000, P.R. China \\ Corresponding author: E-mail: xsc9613017@163.com
}

(Received: 2 April 2012;

Accepted: 29 December 2012)

AJC-12639

A novel three-dimensional $(3 \mathrm{D}) \mathrm{Cd}(\mathrm{II})$ metal-organic frameworks $\left[\mathrm{Cd}_{3}(2,6 \text {-ntd })_{3}(\mathrm{DMA})_{4}\right](1 ; 2,6$-ntd $=2,6$-naphthalene dicarboxylic
acid; $\mathrm{DMA}=N, N^{\prime}$-dimethyl acetamide) has been solvothermally synthesized. Single-crystal structure analysis reveals that this compound
crystallized in a monoclinic space group of $\mathrm{C} 2 / \mathrm{c}$ with a $14.4124(4) \AA, \mathrm{b}=18.5328(4) \AA, \mathrm{c}=21.3181(5) \AA, \alpha=90.00^{\circ}, \beta=98.811(2)^{\circ}$,
$\lambda=90.00^{\circ}, \mathrm{V}=5626.9(2) \AA^{3}$ and $\mathrm{Z}=4$. From the viewpoint of topology, it can be reduced to a 6-connected PCU topological network.
Key Words: Cadmium, Metal-organic framework, PCU topology, 2,6-Naphthalene dicarboxylic acid.

Metal-organic frameworks, in addition to their versatile intriguing topological structures, as a new class of porous materials, have attracted considerable attention during the past decades because of unique properties for potential applications in gas storage and separation, molecular recognition, heterogeneous catalysis, non-linear optics, ion exchange, fluorescence and magnetism, etc. ${ }^{1-5}$. It has been found that organic ligands play crucial roles in the diversification and special characteristics of some target metal-organic frameworks. The 2,6-naphthalene dicarboxylate (2,6-ntd) ligand has been widely used for constructing a metal-organic frameworks porous materials ${ }^{6,7}$. Herein, we report the synthesis of one novel porous metal-organic frameworks constructed adopting 2,6-ntd ligand, namely $\left[\mathrm{Cd}_{3}(2,6-\text { ntd })_{3}(\mathrm{DMA})_{4}\right](\mathbf{1})$.

The chemicals with reagent-grade quality employed were commercially available and used as received without further purification.

Preparation of compound: A mixture of $\mathrm{Cd}\left(\mathrm{NO}_{3}\right)_{2} \cdot 4 \mathrm{H}_{2} \mathrm{O}$ (0.0955 g, $0.31 \mathrm{mmol})$, pyrazine $(0.0178 \mathrm{~g}, 0.22 \mathrm{mmol}), 2,6-$ naphthalene dicarboxylic acid (2,6-ntd, $0.0769 \mathrm{~g} 0.36 \mathrm{mmol})$, 1,5-pentamethylene- $1 H$-tetrazole $(0.0412 \mathrm{~g} 0.30 \mathrm{mmol})$, methanol $(2.0 \mathrm{~mL})$ and $N, N$-dimethylacetamide (DMA, 6.0 $\mathrm{mL}$ ) in a $20 \mathrm{~mL}$ Teflon-lined stainless steel vessel was heated at $120{ }^{\circ} \mathrm{C}$ for $72 \mathrm{~h}$ and then cooled to room temperature. The resulting colourless transparent single crystals of $\mathbf{1}$ suitable for X-ray diffraction were obtained.

X-crystallography: Suitable single crystals were selected under a polarizing microscope and fixed with epoxy cement on fine glass fibers which were mounted on a computercontrolled XCalibur E CCD diffractometer with graphitemonochromated $\mathrm{MoK}_{\alpha}$ radiation $\left(\lambda_{\mathrm{MoK} \alpha}=0.71073 \AA\right)$ at $\mathrm{T}=$ $293.2 \mathrm{~K}$. The hydrogen atoms bound to carbon were located by geometrically calculations. All non-hydrogen atoms were refined by full-matrix least-squares techniques. All calculations were performed by the SHELXTL 97 program $^{8}$.

Structure description: Fig. 1a shows the inorganic building units of 1 that consists of linear trimericmetal centers. The central $\mathrm{Cd}$ atom, $\mathrm{Cd}(2)$, located on an inversion center, is coordinated nearly ideally octahedrally by six carboxylate oxygen atoms. The centrosymmetrically related $\mathrm{Cd}$ atoms in both sides, $\mathrm{Cd}(1)$, are also six coordinated. Their coordination geometry is, however, more irregular and involves four carboxylate oxygen and another two oxygen atoms from DMA. The $\mathrm{Cd}---\mathrm{Cd}$ distance within the trinuclear units is $3.678 \AA$.

Two of the 2,6-ntd ligands have a bridging bis-bidentate coordination mode, nevertheless in the third each carboxylate group has both monodentate bridging and chelating coordination modes to bridge metal atoms (Fig. 1a). The crosslinking of the trinuclear building units are linked via the naphthalene rings to form a 3D-structure (Fig. 1c). A better insight into the nature of this intricate framework can be achieved by the application of a topological approach. As shown in Fig. 1a-c, every nodal point is further linked to result in six-connected frameworks on condition that each trinuclear building units acts as a nodal point, which can be abstracted as a uninodal 6-connected typical PCU topological net. 
(a) $a \underset{\downarrow}{\longrightarrow} \mathrm{c}$

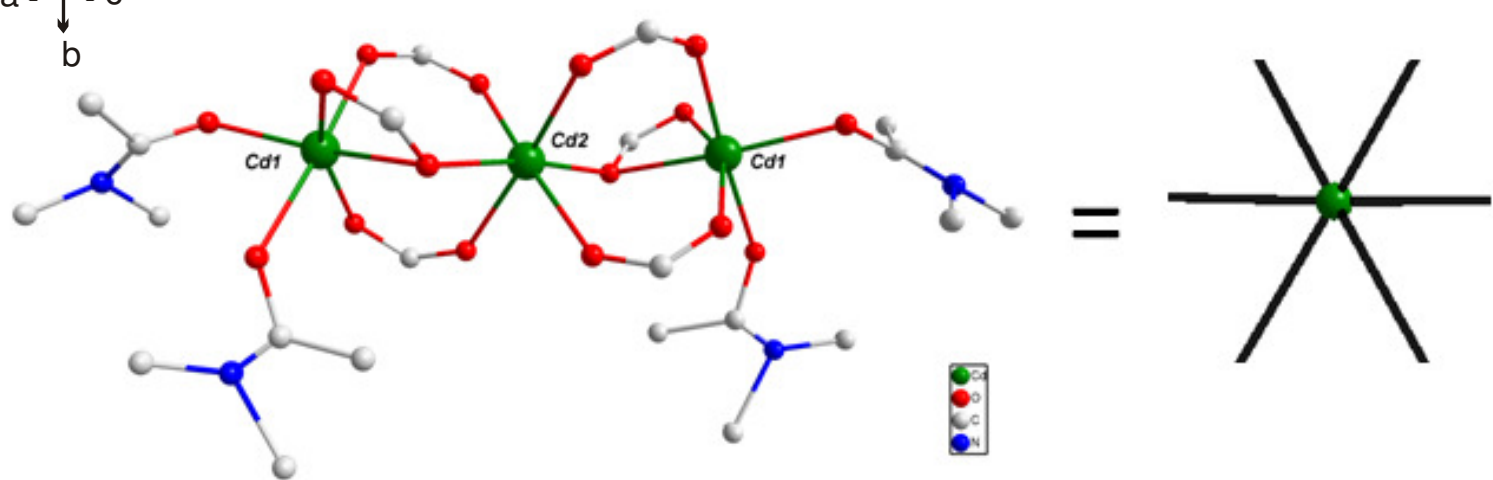

(b)

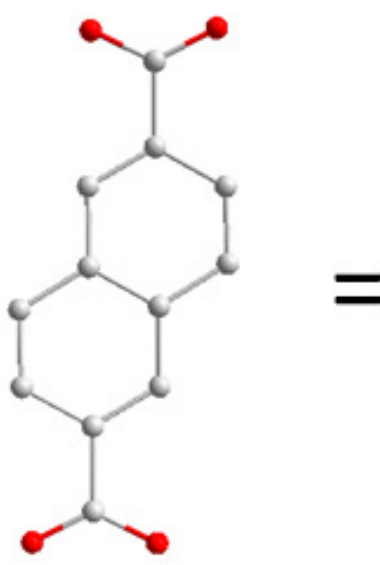

(c)

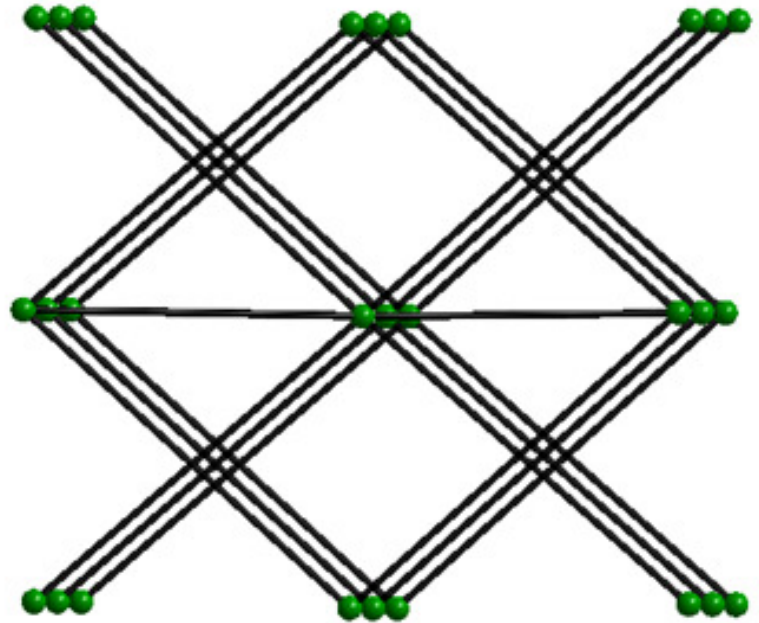

Fig. 1. (a) Trinuclear cadmium unit of $\left[\mathrm{Cd}_{3}(\mathrm{COO})_{6}\right]$ showing a 6-connected node; (b) Schematic diagram of 2,6-ntd; (c) Topological network of 1

\section{REFERENCES}

1. X.M. Zhang, M.L. Tong, H.K. Lee and X.M. Chen, J. Solid State Chem., 160, 118 (2001).

2. S.M. Humphrey and P.T. Wood, J. Am. Chem. Soc., 126, 13236 (2004).

3. Y. Liu, G. Li, X. Li and Y. Cui, Angew. Chem. Int. Ed., 46, 6301 (2007).

4. J.R. Long and O.M. Yaghi, Chem. Soc. Rev., 38, 1213 (2009).

5. Z.Q. Jiang, Asian J. Chem., 25, 2347 (2013).
6. B. Liu, R.Q. Zou, R.Q. Zhong, S. Han, H. Shioyama, T. Yamada, G. Maruta, S. Takeda and Q. Xu, Micropor. Mesopor. Mat., 111, 470 (2008).

7. Q.Y. Liu, W.F. Wang, Y.L. Wang, Z.M. Shan, M.S. Wang and J. Tang, Inorg. Chem., 51, 2381 (2012).

8. G.M. Sheldrick, SHELXTL97, Program for the Refinement of Crystal Structure, University of Gottingen, Germany (1997). 